\title{
Investigation of antioxidant activity of selenium compounds and their mixtures with tea polyphenols
}

\author{
Aleksandra Sentkowska ${ }^{1}$ (1) Krystyna Pyrzyńska²
}

Received: 3 December 2018 / Accepted: 3 March 2019 / Published online: 13 March 2019

(c) The Author(s) 2019

\begin{abstract}
The antioxidant interactions between selenium species and tea polyphenols were investigated using 1,1-diphenyl-2-picryl-hydrazyl (DPPH) radicals, cupric reducing antioxidant capacity (CUPRAC) and Folin-Ciocalteu (FC) assay. Se(IV) exhibited the lowest antioxidant properties in comparison to other selenium compounds in all assays. The highest reducing power was obtained for SeMet, while the highest ability to scavenging DPPH radicals for MeSeCys. The results obtained experimentally for the mixtures containing selenium species and green or black tea infusion were compared with theoretical values calculated by adding up the effects of both individual components analyzed separately. The results obtained from each assay clearly show that observed effect is not additive. In almost every case the theoretical value of antioxidant capacity was significantly higher from that obtained from the activity of the binary mixture of black tea infusion with selenium compound decreased in the order: $\mathrm{SeMet}>\mathrm{Se}(\mathrm{IV})>\mathrm{Se}(\mathrm{VI})>\mathrm{MeSeCys}$, while for similar mixtures with green tea infusion: MeSeCys $>\operatorname{Se}(\mathrm{VI})>$ SeMet $\sim \operatorname{Se}(I V)$.
\end{abstract}

Keywords Selenium compounds · Green tea $\cdot$ Black tea $\cdot$ Antioxidant activity

\begin{tabular}{ll}
\multicolumn{2}{l}{ Abbreviations } \\
BT & Black tea \\
GT & Green tea \\
Se(IV) & Selenite \\
Se(VI) & Selenite \\
MeSeCys & Methylselenocysteine \\
SeMet & Selenomethionine
\end{tabular}

\section{Introduction}

Selenium is widely known as an essential nutrient, which is linked with some serious conditions like cancer, cardiovascular and inflammatory diseases [34]. It plays an important role in many metabolic pathways such as thyroid hormone metabolism and antioxidant defense systems [39]. However, the knowledge of the total selenium amount is not only important, whereas the content of an appropriate selenium

Aleksandra Sentkowska

sentkowska@slcj.uw.edu.pl

1 Heavy Ion Laboratory, University of Warsaw, Pasteura 5A, 02-093 Warsaw, Poland

2 Department of Chemistry, University of Warsaw, Pasteura 1, 02-093 Warsaw, Poland species that is present in particular food or dietary supplements is crucial. It is known that organic forms of selenium are less toxic and more bioavailable than its inorganic forms [22]. Some plants exposed to selenium have ability to transform its inorganic forms into bioactive organic compounds. It has important implications for human nutrition and health as the main source of selenium in human beings is their diet [21, 22, 31, 33]. Selenoaminoacids are widely present in different vegetables such as onion, garlic, broccoli (methylselenocysteine) as well as in rice (selenomethionine) [21, 31].

In the recent years a new potential source of selenium has appeared. One geographically specific selenium-enriched Ziyang tea (Camellia sinensis L.) is widely distributed in the seleniferous region, in Ziyang County, China [10]. The infusion of that green tea in vitro experiments showed higher antioxidant activities in comparison to the regular one [15, 36]. According to Yu et al. [36] the combination of selenium with tea polyphenols was responsible for the observed effects. However, Se-enriched tea has also significantly higher content of phenolic compounds, which are the most abundant antioxidants in the diet [29]. Some selenium compounds or their metabolites can act as antioxidants, prooxidants or both depending of specific conditions [23, 34, 39]. Selenoaminoacids can also provide antioxidant benefits by acting as a source of Se for synthesis of selenium-dependent 
antioxidants and repair proteins such as gluthathione peroxidases, thioredoxin reductases and methionine sulfoxide [23]. From the other side, Tsopelas et al. [32] reported that among several studied selenium compounds only urea derivatives, such as selenourea and dimethylselenourea, exhibited considerable antioxidant properties according to their oxidation potential. The investigation of combining polyphenol and selenium functionalities and preparation several analogues of polyphenolic acid and phenylselenoethanol was reported by Lin et al. [12]. However, the free radical scavenging profiles of these Se-containing polyphenolic acid esters evaluated using DPPH method were lower than analogous caffeic acid ester.

The aim of this study was to evaluate the antioxidant interactions between selected selenium species and tea polyphenols employing three methods: scavenging of the stable 2,2-diphenyl-1-picrylhydrazyl (DPPH) radical, cupric reducing antioxidant capacity (CUPRAC) and Folin-Ciocalteu assay. In particular, we have compared the antioxidant capacity of the mixtures containing green tea or black tea polyphenols and selected selenium species with that of the single components measured individually.

\section{Materials and methods}

\section{Reagents and samples}

The commercial standards of sodium selenate, sodium selenite, selenomethionine, methylselenocysteine as well as the standards of green and black tea infusions were purchased from Merck-Sigma (Steinheim, Germany). Methanol was purchased from Merck (Darmstadt, Germany). Ultra-pure water from Mili-Q system (Milipore, Bedford, MA,USA) with electrical resistivity of $18 \Omega \mathrm{cm}^{-1}$ was used in all experiments. Stock solutions of selenium species, tea standards and their mixtures were prepared in water. Each sample were analyzed in triplicate.

\section{Instrumentation}

The DPPH assay was applied to estimate the radical-scavenging ability of green tea extract (Dróżdż, Sentkowska \& Pyrzyńska, [2]). $0.1 \mathrm{~mL}$ of the appropriate mixture of components or individual compounds was mixed with $2.4 \mathrm{~mL}$ of DPPH solution $\left(9 \times 10^{-5} \mathrm{~mol} / \mathrm{L}\right)$ in methanol. The change of absorbance at $518 \mathrm{~nm}$ was recorded after $30 \mathrm{~min}$. The antioxidant activity was expressed in terms of EC50 value, i.e. the concentration (in $\mu \mathrm{M}$ ) required to scavenge $50 \%$ of DPPH radicals and in terms of trolox equivalent (TEAC).

The cupric ion reducing antioxidant capacity of all extracts was determined according to the method of Özyürek et al. [18]. $1 \mathrm{~mL}$ of $\mathrm{CuCl}_{2}$ solution $(0.01 \mathrm{~mol} / \mathrm{L})$ was mixed with $1 \mathrm{~mL}$ of neocuproine solution $(7.5 \times 10-3 \mathrm{~mol} / \mathrm{L})$ and $1 \mathrm{~mL}$ of $1 \mathrm{~mol} / \mathrm{L} \mathrm{CH}_{3} \mathrm{COONH}_{4}$, followed by adding $0.5 \mathrm{~mL}$ of standard sample and $0.6 \mathrm{~mL}$ of water. The mixture was incubated in a water bath at the temperature of $50{ }^{\circ} \mathrm{C}$ for $20 \mathrm{~min}$. The absorbance against the reagent blank was measured after $30 \mathrm{~min}$ at $450 \mathrm{~nm}$. The results were expressed as trolox equivalent in $\mu \mathrm{M}$.

The Folin-Ciocalteu assay was conducted based on the method described by Sigleton et al. [30] with some changes. $1 \mathrm{~mL}$ of a sample solution was mixed with $0.1 \mathrm{~mL}$ of FC reagent and $0.9 \mathrm{~mL}$ of water. After $5 \mathrm{~min}, 1 \mathrm{~mL}$ of sodium carbonate $(7 \% \mathrm{w} / \mathrm{v})$ and $0.4 \mathrm{~mL}$ of water were added and 30 more min was allowed for stabilization of the blue color formed. The absorbance against blank was measured at $765 \mathrm{~nm}$ and the results were expressed as gallic acid equivalent (GAE) in $\mathrm{mM}$.

\section{Statistical analysis}

The results are from at least three independent experiments and presented as average \pm standard deviation. One-way ANOVA and Turkey test was used to determine the difference of means. Significance was defined at $\mathrm{P}$ values $<0.05$.

\section{Results and discussion}

There are many well-known methods that can be used for study the antioxidant activity [26]. The choice which is appropriate for particular samples should be based on the chemistry of the compounds of interest. Some methods are concerned with electron or radical screening, whereas other assays are focused on reducing ability. In this study three assays differ in the reaction mechanism were used. The antioxidant activity were evaluated using scavenging of a model radical 2,2-diphenyl-1-picrylhydrazyl (DPPH) and cupric reducing antioxidant capacity (CUPRAC). The reducing power of the studied compounds was also evaluated by Folin-Ciocalteu (FC) assay. This method is often called "total phenolic content", but FC reagent is not specific for the phenolic compounds as other compounds give elevated apparent phenolic content $[1,4]$. The chemistry behind FC assay counts on a single electron transfer of electrons in alkaline medium from phenolic compounds and other reducing species to molybdenum, forming blue complexes that can be monitored spectrophotometrically. It is assumed that the antioxidant activity is equal to the reducing power of the sample.

\section{Antioxidant activity of single selenium species}

All measurements were made for the same concentration of the components equals to $10 \mathrm{mg} / \mathrm{L}$. The obtained results 
were expressed by TEAC index (Trolox equivalent antioxidant capacity), which expresses antioxidant capacity of a given substance as equivalent to a certain Trolox concentration (for DPPH and CUPRAC assays) and in GAE index (gallic acid equivalent). The solution of $\alpha$-tocopherol at the same concentration was used for comparison. The results are presented in Table 1. The obtained results in DPPH assay for Se(VI), selenomethinine (SeMet) and methylselenocysteine (MeSeCys) are comparable and only for Se(IV) was significantly lower. $\alpha$-tocopherol showed higher antioxidant activity in comparison to all studied forms of selenium. The EC50 parameters (the effective concentration necessary to reduce $50 \%$ of DPPH radicals) for selenium compounds were also experimentally quantified [24]. Lower value of EC50 parameter means higher antioxidant activity. The results presented in Table 1 confirmed that selenium compounds are weaker antioxidants than $\alpha$-tocopherol. Se(IV) exhibited the lowest ability for scavenging the DPPH radicals, while the highest one was observed for SeMeCys. The results of one-way ANOVA followed by Turkey's test indicated that TEAC index for other selenium species does not vary significantly $(\mathrm{p}>0.05)$.

CUPRAC method is based on reduction of $\mathrm{Cu}(\mathrm{II})$ to $\mathrm{Cu}(\mathrm{I})$ at neutral $\mathrm{pH}$ by antioxidants present in the sample utilizing copper(II)-neocuproine reagent as the chromogenic oxidant [18]. In this assay only $\mathrm{Se}(\mathrm{VI})$ showed higher antioxidant activity than $\alpha$-tocopherol and it was the highest value from all studied compounds (Table 1). In CUPRAC method, similar to DPPH method, Se(IV) exhibits the lower antioxidant properties in comparison to other selenium compounds. The reducing capacity for selenium compounds increases in the order: $\mathrm{Se}(\mathrm{IV})<$ SeMetCys $<\mathrm{Se}(\mathrm{VI})<$ SeMet (Table 1). SeMet is easily oxidized and the first appearing degradation product is selenomethionoine selenoxide, which then is converted via the deaminated selenoxide to methane seleninic acid and selenite [7].

The reducing power of studied compounds and their mixtures was also evaluated by Folin-Ciocalteu assay [30]. Taking into consideration that the FC reagent reacts also with several non-phenolic compounds such as reducing sugars, ascorbic acid, some transition metals and reducing amino acids, this assay has been denominated as "Folin-Ciocalteau reducing capacity" [9]. As can be seen in Table 1, the values for selenium species are much lower than the value obtained for the same concentration of $\alpha$-tocopherol. The reducing capacity for selenium compounds were increased in the order: $\mathrm{Se} \mathrm{IV})<\mathrm{Se}(\mathrm{VI})<$ SeMetCys $<$ SeMet. It can be noticed that $\mathrm{Se}(\mathrm{VI})$ exhibits higher reducing power than SeMetCys in FC assay in comparison to the results obtained in CUPRAC method. Although both used assays are based on the redox properties of studied compounds, but their differ in terms of reduction potentials, kinetics and experimental conditions.

\section{Antioxidant activity of selenium mixtures}

Cultivation of plants enriched with selenium could be an effective way of producing selenium rich foodstuffs and thereby increase health benefits. In these samples selenium can exists, depending on the element form used for enrichment, in inorganic species (Se (IV) and Se (VI)) as well as selenoaminoacids (SeMet and MeSeCys), which are the products of inorganic selenium transformation [21, 22]. Thus, the mixture of different selenium forms can exist in the analyzed extracts. In our study the antioxidant activity of binary mixtures containing selenium species were investigated using previously described assays. The results obtained experimentally were compared with the theoretical values calculated by adding up the effects of individual components at the same concentration analyzed separately. This is commonly used method for study the antioxidant interaction between different kind of compounds [5, 19, 25]. Selenium in natural and food samples can exist in different chemical forms. It should be speculated that they can interact which each other. Such interaction can have the impact on antioxidant properties of whole sample that is analyzed. That is why studying the interaction between its different speciation forms is necessary. The obtained results are presented in Fig. 1.

For every analyzed mixtures in all used assays the calculated theoretical value of antioxidant activity was significantly higher than the corresponding value obtained
Table 1 The antioxidant acitivities of selenium species and $\alpha$-tocopherol

\begin{tabular}{|c|c|c|c|c|}
\hline \multirow[t]{2}{*}{ Compound } & \multicolumn{2}{|l|}{ DPPH } & \multirow{2}{*}{$\begin{array}{l}\text { CUPRAC } \\
\text { TEAC }(\mu \mathrm{M})\end{array}$} & \multirow{2}{*}{$\begin{array}{l}\text { FC } \\
\text { GAE }(\mathrm{mM})\end{array}$} \\
\hline & TEAC $(\mu \mathrm{M})$ & $\mathrm{EC}_{50}(\mu \mathrm{M})$ & & \\
\hline $\mathrm{Se}(\mathrm{IV})$ & $65.02 \pm 3.2^{\mathrm{a}}$ & $1.36 \pm 0.07^{\mathrm{a}}$ & $8.75 \pm 0.43^{\mathrm{a}}$ & $0.41 \pm 0.020^{\mathrm{a}}$ \\
\hline $\mathrm{Se}(\mathrm{VI})$ & $216.0 \pm 10.8^{b}$ & $0.98 \pm 0.04^{b}$ & $114.2 \pm 5.4^{\mathrm{b}}$ & $3.93 \pm 0.196^{b}$ \\
\hline SeMet & $215.1 \pm 10.1^{\mathrm{b}}$ & $1.00 \pm 0.04^{\mathrm{b}}$ & $181.0 \pm 9.0^{\mathrm{c}}$ & $4.43 \pm 0.221^{\mathrm{c}}$ \\
\hline MetSeCys & $223.2 \pm 11.0^{\mathrm{b}}$ & $0.30 \pm 0.02^{c}$ & $109.4 \pm 5.4^{b}$ & $2.33 \pm 0.116^{\mathrm{d}}$ \\
\hline$\alpha$-tocopherol & $294.03 \pm 8.3^{\mathrm{c}}$ & $0.15 \pm 0.03^{\mathrm{d}}$ & $141.2 \pm 7.2^{\mathrm{c}}$ & $20.3 \pm 1.01^{\mathrm{e}}$ \\
\hline
\end{tabular}

Concentration of all compounds was $10 \mathrm{mg} / \mathrm{L}$. Values in each column bearing different superscript letters represent significant differences $(p<0.05$, ANOVA test) 

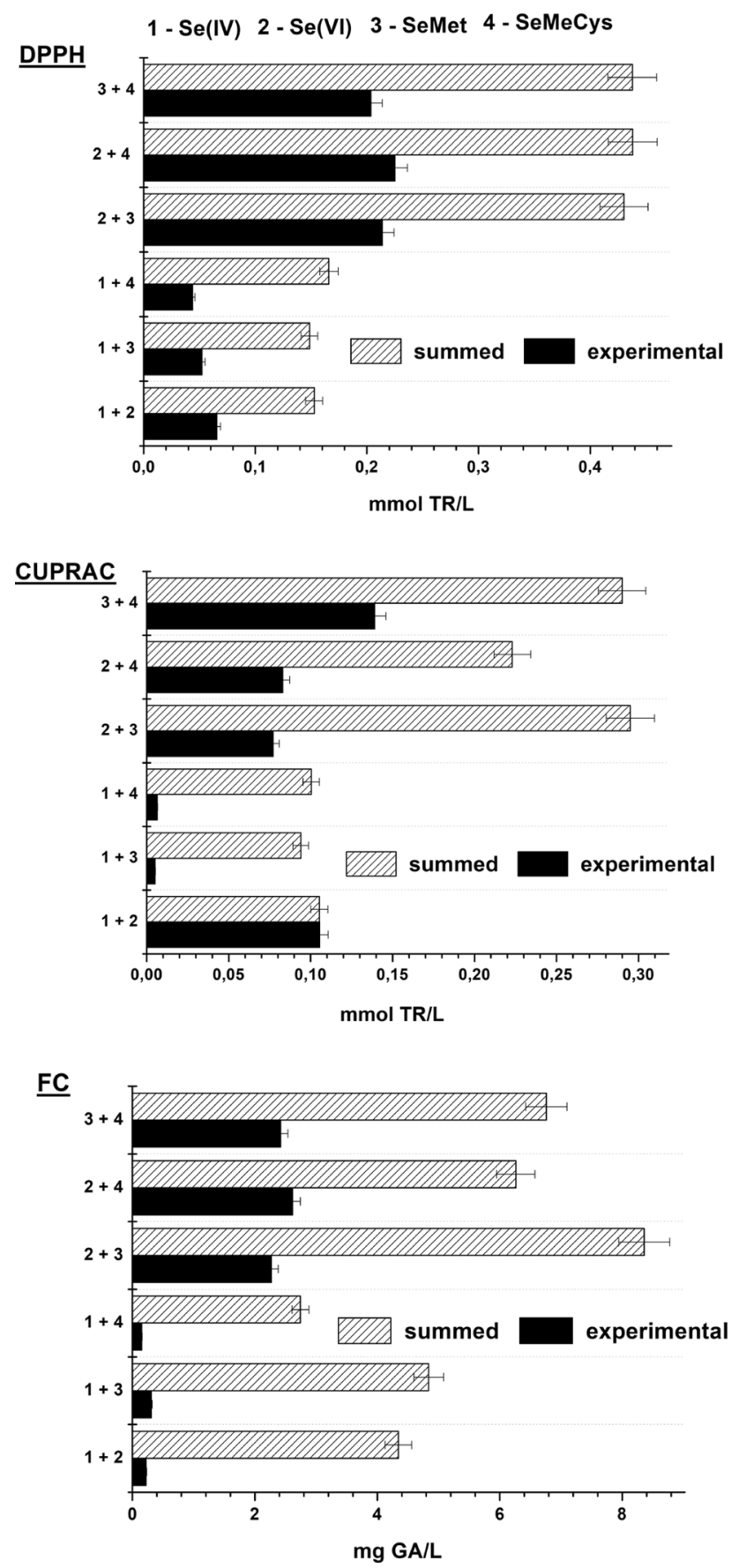

Fig. 1 Antioxidant activity of binary mixtures containing selenium compounds obtained using DPPH, CUPRAC and FC assays. Each compound at concentration $10 \mathrm{mg} / \mathrm{L}$

from experiments. The presence of $\mathrm{Se}(\mathrm{IV})$ in the mixtures with SeMet and MeSeCys significantly decreased their antioxidant activity. This negative effect is most visible in CUPRAC and FC assays. Only the value in CUPRAC assay obtained experimentally for the mixture containing both inorganic selenium species and the theoretical value have no significant difference ( $p>0.05$ ). To the best of our knowledge there no reports about the results for individual selenium species as well as their mixtures in the antioxidant activity assays used in our studies. More studies are needed in order to clarify the observed effects, particularly a dose response at various concentrations.

\section{Antioxidant activity of selenium compounds in the mixtures with tea infusions}

Recently, the research interest have been focused on Seenriched plants which could be used to supplement the human diet owing to its beneficial effect for human health $[21,22,33]$. Tea is the one of the most widely consumed beverages in the world with high content of polyphenolic compounds $[6,14,28]$. Antioxidative properties of these compounds are manifested particularly by their abilities to scavenge free radicals, inhibit their generation, chelate transition metal ions as well as to increase the activity of enzymes with antioxidative properties [13, 27]. There are some reports that tea supplemented in different ways (Se-enriched fertilizer, foliar spraying) exhibited significantly higher antioxidant activity than regular tea $[15$, 36-38]. It was reported that Se-enriched tea and regular tea exhibited EC50 values equals to $22.43 \pm 0.13 \mathrm{mg} / \mathrm{L}$ and $23.65 \pm 0.13 \mathrm{mg} / \mathrm{L}$, respectively [36]. According to Molan et al. [15] for Se-enriched tea the DPPH free-radical scavenging percentage was $74.2 \pm 0.85 \%$ and the value of $72.4 \pm 0.18 \%$ was obtained for regular China tea. The antioxidant activity of the tea extract leaves in DPPH assay decreased in the order: tea obtained by fertilization by $\mathrm{Se}(\mathrm{VI})>$ fertilization by $\mathrm{Se}(\mathrm{IV})>\alpha$-tocopherol $>$ regular tea [20]. Information regarding the comparison of phenolic content in these samples, which mainly contributed to the antioxidant activity of tea, are conflicting. Lower [36], higher [15] or similar [38] value have been reported.

On the other hand, there is well known that polyphenols as well as other bioactive compounds could interact between each other, which is clearly visible in the antioxidant activities of their mixtures [3, 8, 11, 20]. Mouly et al. [17] reported that over 300 remedies and mineral supplements are capable of interacting with food components through essential mineral antagonism, induction of inhibition of micronutrient metabolism. It can be predicted that also interactions between the polyphenols and selenium compounds may occur. Thus, in this study the effect of the addition of different selenium species to the samples of green (GT) and black (BT) tea infusions on their antioxidant activity was evaluated. The kinetic curves of scavenged DPPH radicals by BT and GT aqueous extracts and their binary mixtures with appropriate selenium species are presented in Fig. 2.

A fast initial decrease of DPPH absorbance followed by slow subsequent disappearance of this reagent can be observed from the presented plots. According to Villaño 
(a)
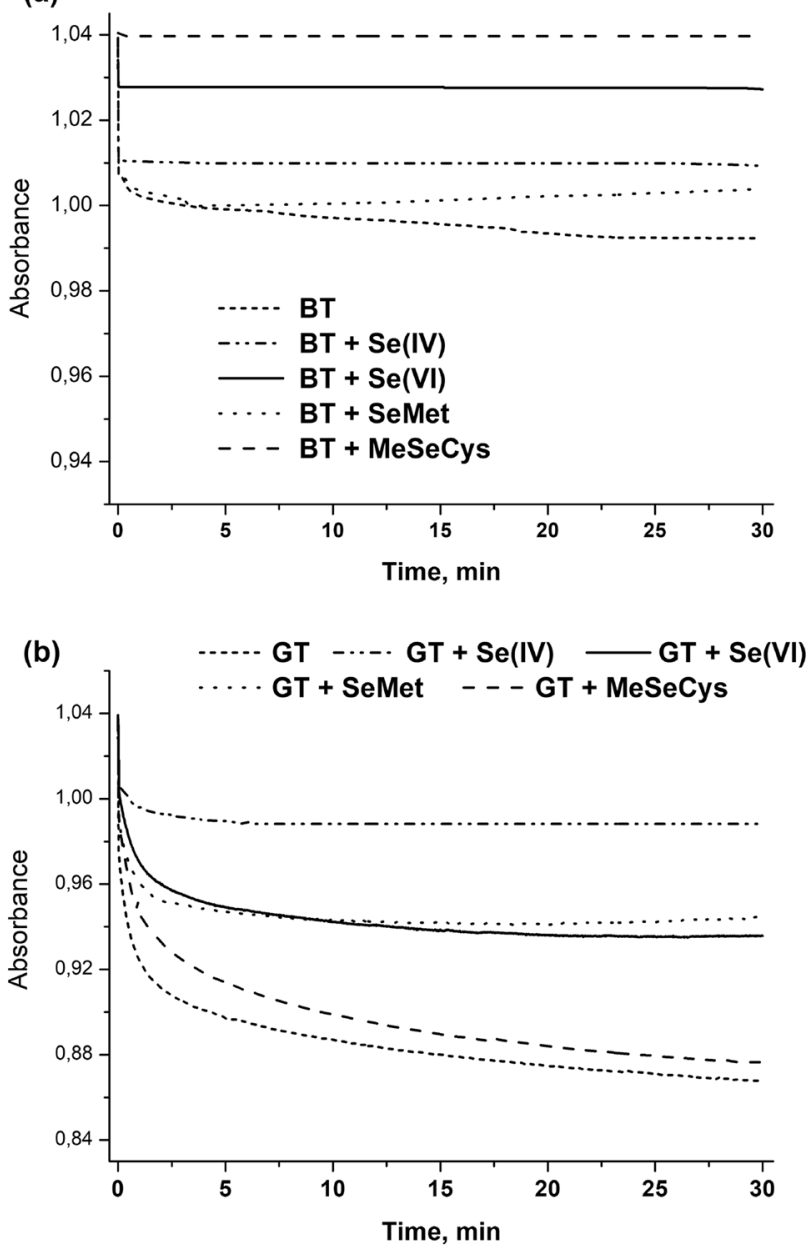

Fig. 2 The kinetic curves of scavenged DPPH radicals by a black tea (BT) b green tea (GT) infusions and their mixtures with selenium species. Concentration of each Se compounds equals to $10 \mathrm{mg} / \mathrm{L}$

et al. [35], this fast step essentially refers to the electrontransfer process from a phenol molecule or its phenoxide anion to DPPH free radical, while the subsequent decay reflects the remaining activity of the oxidation-degradation products. In every case, the binary mixture of selenium compound and tea polyphenols showed lower antioxidant properties in comparions to alone tea infusion. However, different behaviour of black and green tea extracts in the presence of a given selenium compounds were observed. While MeSeCys decreased the antioxidant activity of BT almost to zero, the presence of this selenoaminoacid in the mixture with GT did not change the plot recorded for black tea infusion alone. The antioxidant activity of the binary mixture of black tea infusion with Se compound decreased in the order:

SeMet $>\mathrm{Se}(\mathrm{IV})>\mathrm{Se}(\mathrm{VI})>\mathrm{MeSeCys}$, while this order for similar mixtures with green tea infusion was: MeSeCys $>\mathrm{Se}(\mathrm{VI})>\mathrm{SeMet} \sim \mathrm{Se}(\mathrm{IV})$. The results of one-way ANOVA followed by Turkey's test indicated that TEAC index for BT mixtures with SeMet and Se (IV) does not vary significantly $(p>0.05)$. On the other hand such mixtures vary significantly when GT instead BT is used $(\mathrm{p}<0.05)$. The same observation was made for the tea itself and its mixture with MeSeCys- the result is not statistically significant $(\mathrm{p}>0.05)$ when GT is used. The composition of green and black tea infusions differ from each other in terms of polyphenolic content as well as presence of other substances [14, 27]. The monomeric catechins and their derivatives are the major polyphenols in green tea. Black tea receives substantial oxidation, which results in the polymerization of catechins into theaflavins and thearubigins. There is the possibility that tea polyphenols and Se species form molecular complexes with antioxidant activity different from the activity of individual components. Moreover, these observed effects could also depend on the different ratios of bioactive components in the mixtures as well as the antioxidant mechanisms of these phytochemicals. Motomura et al. [16] found that the relative radical scavenging activity in DPPH assay of selenite-treated red clover extract decreased slightly, up to $0.2 \mathrm{mM}$ of $\mathrm{Se}(\mathrm{IV})$, and then decreased strongly (about 79\%) but in ryegrass extract no significant difference was observed with the increase of selenite content. Thus, further investigations are necessary to adress the function of phenolic antioxidative activity considering the beneficial effects of these compounds and selenium species. Isobolographic analysis, which take into account of relative dose of components, will be further used in the evaluation of antioxidant interactions.

\section{Conclusions}

These studies demonstrate the complex interactions between the different speciation forms of selenium and tea components. These interactions may have a significant influence on the mixture antioxidant activity. These results may be important from the point of view of supplementation of tea trees with selenium. More studies are needed to be performed for better describing the tea-selenium antioxidant interactions.

Acknowledgements Authors gratefully acknowledges that this work was financially supported by National Science Centre, Poland (MINIATURA 2017/01/X/NZ9/01521).

\section{Compliance with ethical standards}

Competing interests The authors declare that there is no conflict of interest regarding the publication of this paper.

Open Access This article is distributed under the terms of the Creative Commons Attribution 4.0 International License (http://creativeco mmons.org/licenses/by/4.0/), which permits unrestricted use, distribution, and reproduction in any medium, provided you give appropriate credit to the original author(s) and the source, provide a link to the Creative Commons license, and indicate if changes were made. 


\section{References}

1. Castro-Alves VC, Cordenunsi BR (2015) Total soluble phenolic compounds quantification is not as simple as it seems. Food Anal Methods 8:873-884

2. Dróżdż P, Sentkowska A, Pyrzynska K (2017) Flavonoid content and antioxidant properties in different extracts of Calluna vulgaris (L.) flowers. J Agric Sci Technol A 7:39-44

3. Enko J, Gliszczyńska-Świgło A (2015) Influence of the interactions between tea (camellia sinensis) extracts and ascorbic acid on their antioxidant activity: analysis with interaction indexes and isobolograms. Food Addit Contam A 32:1234-1242

4. Everette JD, Bryant QM, Green AM, Abbey YA, Wangila GW, Walker RB (2010) Through study of reactivity of various compound classes toward the Folin-Ciocalteu reagent. J Agric Food Chem 58:8139-8144

5. Freeman BL, Egget DL, Parker TL (2010) Synergistic and antagonistic interactions of phenolic compounds found in navel oranges. J Food Sci 75:C150-C176

6. Finley JW, Kong AH, Hintze KJ, Jeffery EH, Ji LL, Lei XG (2011) Antioxidants in foods: state of the science important to the food industry. J Agric Food Chem 59:6837-6846

7. Gammelgaard B, Cornett C, Olsen J, Bendahl L, Hansen SH (2003) Combination of LC-ICP-MS, LC-MS and NMR for investigation of the oxidative degradation of selenomethionine, Talanta. $59,1165-1171$

8. Gawlik-Dziki U (2012) Changes in the antioxidant activities of vegetables as a consequence of interactions between active components. J Funct Foods 4:872-882

9. Granato D, Santos JS, Maciel LG, Nunes DS (2016) Chemical perspective and criticism on selected analytical methods used to estimate the total content of phenolic compounds in food matrices. TRAC Trends Anal Chem 80:266-279

10. He X, Li J, Zhao W, Liu R, Zhang L, Kong X (2015) Chemical fingerprint analysis for quality control and identification of Ziyang green tea by analysis. Food Chem 171:405-411

11. Heo HJ, Kim YJ, Chung D, Kim DO (2007) Antioxidant capacities of individual and combined phenolics in a model system. Food Chem 104:87-92

12. Lin CF, Chang TC, Chang CC, Tsal HJ, Hsu LH (2005) Synthesis of selenium-containing polyphenolic acid esters and evaluation of their effects on antioxidation and 5-lipoxygenase inhibition. Chem Pharm Bull 53:1402-1407

13. Lorenzo JM, Munekata PES (2016) Phenolic compounds of green tea: health benefits and technological application in food. Asian Pac J Trop Biomed 6:709-719

14. Mojzer EB, Hrnčič M, Knez Ž, Bren U (2016) Polyphenols: extraction methods, antioxidative action, bioavailability and anticarcinogenic effects. Molecules 21:901-939

15. Molan AL, Flanagan J, Wei W, Moughan PJ (2009) Selenium containing green tea has higher antioxidant and prebiotic activities than regular green tea. Food Chem 114:829-835

16. Motomura Y, Reyes-Diaz M, de la LM Gil, M (2008) Effect of selenite on the totaol polyphenol content and antioxidative activity of aqueous and ethanolic extracts in sprouts of four agronomic species. J Soil Sci Plant Nutr 8:55-67

17. Mouly S, Lloret-Linares C, Sellier PO, Sene D, Bergmann JF (2017) Is the clinical relevance of drug-food and drug-herb interactions limited to grapefruit juice and Saint-John`s Wort. Pharmacol Res 118:82-92

18. Özyürek M, Güçlü K, Tütem E, Başkan K, Erçag SE, Baki S, Vildiz L, Karaman S, Apak R (2011) A Comprehensive review of CUPRAC methodology. Anal Methods 3:2439-2453

19. Palafox-Carlos H, Gil-Chaves J, Sotelo-Mundo RR, Namieśnik J, Gorinstein S, Gonzales- Aguilar A (2012) Antioxidant interaction between major polyphenolic compounds found in "Ataulfo" mango pulp: chlorogenic, gallic, protocatechuic and vanilic acids. Molecules 17:12657-12664

20. Pinelo M, Manzoccom L, Nunezm MJ, Nicoli MC (2004) Interaction among phenols in food fortification: negative synergism and antioxidant capacity. J Agric Food Chem 52:1177-1180

21. Pyrzyńska K (2009) Selenium speciation in enriched vegetables. Food Chem 114:1183-1191

22. Pyrzyńska K (2014) Edible plants enriched with selenium. J Agric Sci Technol A 4:627-632

23. Rahmanto AS, Davies MJ (2012) Selenium-containing amino acids as direct and indirect antioxidants. Life 64:863-871

24. Sebaugh JL (2011) Guidelines for accurate EC50/IC50 estimation. Pharm Stat 10:128-134

25. Sentkowska A, Pyrzyńska K (2018) Zwitterionic hydrophilic liquid chromatography coupled to mass spectrometry for analysis of beetroot juice and antioxidant interactions between its bioactive compounds. LWT 93:641-648

26. Shahidi F, Zhong Y (2015) Measurement of antioxidant activity. J Funct Foods 18:757-781

27. Shahidi P, Ambigaipalan P (2015) Phenolics and polyphenolics in food, beverages and spices: antioxidant activity and health effects-a review. J Funct Foods 15:820-897

28. Sharangi AB (2009) Medicinal and therapeutic potentialities of tea (Camelia sinesis L.). A review. Food Res Int 42:529-535

29. Sies H (2010) Polyphenols and health: update and perspective. Arch Biochem Biophys 501:6-9

30. Singleton VI, Orthofer R, Lamuela-Ravwntos RM (1999) Analysis of total phenolics and other oxidation substances and antioxidants by means of Folin-Ciocalteu reagent. Methods Enzymol 299:125-178

31. Sun GH, Li X, Williams PN, Zhu YG (2010) Distribution and translocation of selenium from soil to grain and its speciation in paddy rice (Oryza sativa L.). Environ Sci Technol 44:6706-6711

32. Tsopelas F, Tsantili-Kakoulidou A, Ochsenkühn-Petropolou M (2013) Lipophilicity, biomimetic retention profile and antioxidant activity of selenium species. Michrochem J 110:711-718

33. Wan J, Zhang M, Adhikari B (2018) Advances in seleniumenriched foods: from the farm to the fork. Trends Food Sci Technol 76:1-5

34. Weekley CM, Harris HH (2013) Which form is that? The importance of selenium speciation and metabolism in the prevention and treatment of disease. Chem Soc Rev 42:870-8894

35. Villaño D, Fernández-Pachón MS, Moyá ML, Troncoso AM, García-Parrilla MC (2007) Radical scavenging ability of polyphenolic compounds towards DPPH free radical. Talanta 71:230-235

36. Yu F, Sheng J, Xu J, An X, Hu Q (2007) Antioxidant activities of crude tea polyphenols, polysaccharides and proteins of seleniumenriched tea and regular tea. Eur Food Res Technol 225:843

37. Xu J, Yang F, Chen L, Hu Y, Hu Q (2003) Effect of selenium on increasing the antioxidant activity of tea leaves harvested during the early spring tea producing season. J Agric Food Chem 51:1081-1084

38. Xu J, Zhu S, Yang F, Cheng L, Hu Y, Pan G, Hu Q (2003) The influence of selenium on the antioxidant activity of green tea. J Sci Food Agric 83:451-455

39. Zimmerm MT, Bayse CA, Ramoutar RR, Brumaghim JL (2015) Sulfur and selenium antioxidants: challenging radical scavenging mechanism and developing structure-activity relationship based on metal binding. J Inorg Biochem 145:30-40

Publisher's Note Springer Nature remains neutral with regard to jurisdictional claims in published maps and institutional affiliations. 